\title{
The Religious Schizophrenic: Why Spirituality is Crucial for Recovery
}

\author{
By Emily Sweet
}

\begin{abstract}
Up to $80 \%$ of schizophrenic patients use religion to cope with their illness. These positive spiritual coping strategies are the primary predictor of mental wellness in patients with schizophrenia. Yet, most medical professionals have no religious training and are often ill-equipped to guide their schizophrenic patients in spiritual matters. Typically, religious institutions and modern medicine are not associated together, but what happens when mental health professionals lack the training to assist $80 \%$ of their schizophrenic patients who use religion as a coping strategy? Schizophrenic patients whose beliefs are not respected have a higher rate of suicide, face increased stigma and report a lower overall quality of life. Such patients are more likely to decline mentally and drop out of treatment. Some scholars, psychologists and philosophers are now arguing that ignoring the connection between religion and mental wellness is unethical because practitioners are failing to take patient diversity into account. This paper will attempt to answer the following questions: In an increasingly diverse world, is it the responsibility of mental health professionals to learn about their patients' religious beliefs, especially when their beliefs are so closely intertwined with their chances at successfully managing their illness, such as the case with schizophrenics? Why is it a good idea to consider combining religion and healthcare? Should the increase in diverse patients require additional training for mental health professionals? Is it unethical for a mental health care professional to be ignorant of diverse cultures and religions? What are the dangers of allowing medical professionals, who largely have no training in religious affairs, to guide mentally ill patients? What are the potential solutions for this problem? Which solutions are more effective and why? Are the current practiced healthcare models, which combine medicine and religion, effective?
\end{abstract}

Keywords: schizophrenia, religion, treatment, therapy, psychosis, stigma

\section{Background}

"One-third of patients with schizophrenia are very highly involved in religious community" and "studies suggest that up to $80 \%$ of patients use religious coping as a means of dealing with their illness," which means that religion is an important aspect for many schizophrenics (Chakrabarti et al. 2014, pp. 119, 121). Religion has shown to be an effective coping strategy for people with schizophrenia, "for example, positive coping mechanisms such as petitionary prayer and making helpful religious attributions have been useful to patients for reducing distress, social alienation, anxiety, and non-adaptive behaviors related to the presence of positive psychotic symptoms...it also appears to protect against suicide attempts and enhance life satisfaction" (Escamilla et al. 2014, p. 1623).

Religious beliefs are so important to a large percentage of schizophrenics. There is evidence to suggest that religious communities provide benefits for people with schizophrenia, as outlined above. Mental health professionals,

"Guest Lecturer, York University \& Humber College, Canada. 
therefore, need to start acknowledging religion as an important factor for treatment and recovery in their schizophrenic patients. Unfortunately, there are many barriers which prevent people with schizophrenia from fully benefitting from the positive side effects of being involved in religion.

This paper will aim to explore the connection between schizophrenia and religion to bring attention to a seldomly discussed topic in medical science: the connection between religion and mental wellness. Underestimating this important connection can lead to grave consequences for schizophrenic patients, including suicide (Prout et al. 2016, p. 6). Doctors do not learn about religion in medical school, yet the research outlined in this paper shows a clear link between mental wellness in schizophrenics and religion. As a result, there is a lack of "culturally competent practitioners," who are able to grasp how their patients' mental illnesses are impacted by their religion and culture (Dempsey et al. 2016, p. 76). One solution to the lack of education about the importance of religion on patient wellness is integrated healthcare. This system allows for education and collaboration between religious leaders and mental health professionals. If healthcare practitioners fail to acknowledge and work with their patients' religious beliefs, then they are doing a disservice to schizophrenics who greatly benefit from religious involvement. To achieve these objectives, this paper will review related literature, studies and medical care models, which will be largely sourced from JSTOR.

\section{Discussion}

\section{Social Stigmatism as a Barrier to Treatment}

One of the health barriers schizophrenics face is social stigmatism. Many Asian countries, including China, Thailand, Japan and Singapore, for example, hold negative social stereotypes about people with schizophrenia, such as considering them "violent," "annoying" or having a "a weak personality" (Zhang et al. 2019, p. 7). Social stigma negatively impacts schizophrenic patients' quality of life, self-esteem, adherence to treatment and even their family's social status and their employment in the community (Zhang et al. 2019, p. 4). This social stigma leads to self-stigma — where schizophrenics negatively judge themselveswhich "was associated with weaker social support, interaction, and functioning, which in turn affected their symptoms and recovery" (Zhang et al. 2019, p. 6).

Asian-Americans are "disproportionately affected by higher levels of selfstigma" (Cerully et al. 2016, p. 2). This shows that the way cultures view mental illness, can have a direct impact on the patient, even after the person has integrated into a new culture. If schizophrenics are too ashamed to seek help because they fear social reprisal or stigmatization, they often try to deal with their illnesses by themselves - even going so far to deny their diagnosis or claim to have spontaneously recovered (Zhang et al. 2019, 10). Unfortunately, the delay in or outright refusal to seek treatment due to social stigma can lead to more problems 
and is connected to a lower quality of life for schizophrenic patients, as mentioned above.

\section{The Ramifications of Invalidating Religious Experience}

If and when schizophrenics are able to push past these social stigmas to get help, they are met with a new challenge: their religious ideals, which as shown above can have a positive effect on their recovery, are not taken seriously. This happens for two main reasons: The first reason is that "the notion of some basic disorder of higher functions has often encouraged a pessimistic attitude about making sense of what schizophrenics have to say; supposedly, such patients lack the kind of rationality that may be a prerequisite to meaningful speech and dialogue" (Sass 2001, p. 252). Since schizophrenics are shown to "perform poorly on a wide variety of cognitive and perceptual tasks," their lived experiences are not taken seriously by mental health professionals (Sass 2001, p. 252). Instead, they are seen as irrational and are treated accordingly.

When mental health professionals take their religious views seriously, however, schizophrenics are more likely to have a "better recovery and reduced relapse rate," but when their religion is met with negativity, their chance of suicide increases and they are more likely to have a "lower quality of life and higher distress" (Chakrabarti et al. 2014, pp. 120, 121). There seems to be overwhelming evidence that fostering a positive space for schizophrenics - such as encouraging a treatment plan that validates their religious experience-leads to a better quality of life. Many of the negative side effects of social stigma listed above-including social isolation and refusal to seek treatment - are improved when schizophrenics' religious beliefs are taken seriously by mental health professionals (Escamilla et al. 2014, p. 1623).

The second reason that schizophrenics' religious ideas are not taken seriously by mental health professionals is because there is a historical rift between the Catholic Church and the mental health profession. This rift dates back the seventeenth century, when the Catholic Church began using "the testimony of physicians in order to render judgments in cases of blasphemy and heresy" (Allen and Wootton 1983, p. 212). Yet the psychoanalysis of churchgoers did not stop at heretics. Psychiatrists began to criticize all aspects of the Church, which was not received favorably by the Catholic Church. This caused a divide between the Church and the mental health profession and this rift "remains essentially unchanged" to this day (Allen and Wootton 1983, p. 212).

As a result, some mental health professionals still claim that schizophrenics are too cognitively impaired to construct sound religious ideas. These professionals sometimes refuse to positively acknowledge the proven benefits of incorporating their schizophrenic patient's religious beliefs in medical treatment (Chakrabarti et al. 2014, p. 122). Therefore, many schizophrenics are denied the opportunity to use their religion to assist them in their recovery. As mentioned above, when their beliefs are received negatively, schizophrenics are more likely to decline. 


\section{Religious Beliefs as a Marker for a Patient's Mental State}

Not only is it important for mental health workers to acknowledge a schizophrenic's religious belief, so the patient can reap the benefits of religious participation, but trying to understand these beliefs can reveal early warning signs of certain psychotic symptoms. For example, if a schizophrenic is not taken seriously they are more likely to drop out of treatment and their religious beliefs and hallucinations are then left unchecked (Escamilla et al. 2014, p. 1629). This is a problem because without guidance from a mental health professional, religious delusions can get out of control, leading to more severe hallucinations, worsening depressive symptoms and lower psychosocial functioning (Escamilla et al. 2014, p. 1630). Also, the chance that a patient or his or her family will refuse treatment in order to deal with the illness using solely religion also increases when a mental health professional refuses to acknowledge the patient's beliefs (Escamilla et al. 2014, p. 1630).

Another notable factor that is important for mental health professionals to understand is the stages of religious hallucinations and their impact on schizophrenia. When a so-called normal person has a religious experience, many people accept it as a fact. When a schizophrenic person has a religious experience, however, they are seen as delusional. Yet this is not always the case. A psychologist named Christensen created a theory outlining the stages of dramatic religious conversion. He wanted to make sense of the differences between religious conversions in so-called normal people and in patients with schizophrenia. He outlines the stages as follows: "Stage 1: Specific unconscious conflict within a framework of religious belief Stage 2: Conscious conflict related to unconscious conflict, producing guilt, anxiety, and depression Stage 3: Acute reaction precipitated by intensification of the above Stage 4: Withdrawal accompanied by sense of estrangement and often a feeling of unreality Stage 5: Feeling of submission, of giving up or giving to" (Allen and Wootton 1983, p. 216). Interestingly, these stages are all experienced both by schizophrenics experiencing psychosis and by a so-called normal religious convert in a "similar or even identical" manner, up until stage 5 (Allen and Wootton 1983, p. 214).

After stage 5, according to Christensen, there are four ways that the religious conversion experience can impact an individual's mental health based on how their ego or consciousness is impacted after said event. The first two ways are beneficial for mental health: either the person's consciousness doesn't become sharper but their religious experience impacts their life for the better, such as adding a deeper meaning to their life, or they undergo a marked mental and physical transformation, which has a profound positive impact on their psyche. The second two ways are detrimental to mental health: the patient's awareness deteriorates, for example they lose touch with reality to some extent, or there is no rational awareness left. The last two stages are often seen after delusional psychotic episodes and are not actually caused from religious experience (Allen and Wootton 1983, p. 219).

It is interesting to note that the difference between a religious conversion in a healthy-minded individual and a schizophrenic person experiencing a negative, 
psychotic episode is only able to be understood by assessing their mind after the experience, according to Christensen. He claims that conversion, whether gradual or sudden, "is not optional for the truly religious person" (Allen and Wootton 1983, p. 213). Therefore, it can be assumed that conversion experiences are common if they are prerequisite for religious belief and a large percentage of the world is religious to some degree. Also, it could be argued that many of the socalled visions in religious literature could also be dubbed hallucinations, which may or may not have arisen from psychotic episodes. Take the example of when an angel comes to Joseph to tell him that Mary was carrying Jesus (Biblica n.d., 18-25 NIV). Why, then, do millions of Christians find this story holy, while a large amount of mental health professionals could likely diagnose a modern Joseph with a psychotic episode, especially if he was already diagnosed as a schizophrenic?

Understanding the ways in which religious hallucinations manifest and how to determine whether a schizophrenic has experienced a positive, "real" religious experience or a dangerous psychotic episode is crucial for health care providers to understand. Self-proclaimed religious experiences for schizophrenic individuals are a double-edged sword when it comes to treatment.

\section{Religion as a Coping Strategy}

If a schizophrenic patient has a religious experience that results in positive changes to their mental well-being, then denying them the right to benefit from their religious affiliations and beliefs has a detrimental impact on their treatment. Apparently, for example, "positive religious coping" strategies, in one study, was cited as "the primary predictor of psychological well-being" in patients with schizophrenia (Chakrabarti et al. 2014, p. 122). As mentioned above, the social stigmatization of people with schizophrenia often causes feelings of social isolation, which can result in an increase of symptoms related to the illness. Having a community where a patient feels accepted, then, can largely improve their quality of life and can be seen a positive coping strategy.

"Negative religious coping," for example when a patient thinks a demon is after them during a psychotic episode, has "been associated with lesser well-being and adjustment... lower quality of life and higher distress" (Chakrabarti et al. 2014, p. 123). Negative religious coping can also lead to what Christensen considers the two negative potential mental outcomes of a "false" religious conversion experience, which is really a negative psychotic episode and not a religious experience (Allen and Wootton 1983, p. 219). According to Christensen, when a schizophrenic is in one of these states, they lose some or all of their ability to rationally experience the world (Allen and Wootton 1983, p. 219).

When mental health practitioners try to separate religion and psychological treatment, they miss out on the both the potential warning signs that arise from false religious experiences, which "can lead to higher risk of suicide attempts," and the positive impacts of non-psychotic religious experiences, such as a sense of community (Chakrabarti et al. 2014, p. 121). For a doctor to not take seriously a patient's religious beliefs, then, is to miss out on a well of diagnostic information and coping strategies that have been proven to benefit people with schizophrenia. 
"Mental health specialists have a responsibility, therefore, not to dismiss, but to attempt to understand the religious life of their patients" since "religious experience, however distorted," influences a person with schizophrenia (Allen and Wootton 1983, p. 220).

\section{The Stigma of Validating Religious Experiences in Schizophrenia Patients}

When an average person is not aware that an individual has been diagnosed with a mental illness, they are "less likely to identify the mental illness" but "their responses were characteristically negative when labeling was authoritatively assigned," such as by a doctor (Ng 1997, p. 383). Therefore, one could conclude that when a person is labeled with a diagnosis of schizophrenia, people may be more likely to dismiss their religious experience as made up or inaccurate. Most if not all religious experience is based on empirical experience. It is somewhat ironic, therefore, to deny the truth of a schizophrenic's religious experiencesimply due to their diagnosis - because no religious experience can be objectively proven. When someone, such as Joseph in the Bible, is upheld as a sane, holy individual by a large amount of people, the average person would be less likely to identify him as mentally ill than if he was labeled as such, according to the study cited above. How different would the world be if society labeled Joseph as a schizophrenic (or the rough equivalent in his time)? Therefore, "religious beliefs cannot be considered automatically sick or healthy, nor can they be ignored as irrelevant" (Allen and Wootton 1983, p. 219).

Many mental health workers fail to understand and appreciate the religious experiences of their patients with schizophrenia. This often occurs due to social stigma, including the stigma that mental health workers project onto their patients. For example, "around $35.2 \%$ of Chinese mental health professionals admitted a discriminating attitude against psychiatric patients" and "psychiatric inpatients with higher levels of perceived stigma reported lower levels of quality of life" (Zhang et al. 2019, pp. 4-5). There is a direct correlation between discrimination and the severity of schizophrenic symptoms.

Data also shows that when stigma is reduced, people with schizophrenia are more likely to seek treatment, they also report higher levels of "self-esteem and optimism" and are less likely to try to commit suicide (Zhang et al. 2019, p. 15). For example, the Japanese word for schizophrenia, "Seishin-Bunretsu-Byo" was considered "humiliating" (Zhang et al. 2019, p. 7). When this word was changed in 2004 to a more positive label, more schizophrenics sought treatment and reported their diagnosis (Zhang et al. 2019, p. 7). This strongly suggests that reducing both social and medical stigma has a direct correlation on the well-being of people with schizophrenia.

Unfortunately many people with schizophrenia are seen as incompetent, weak or delusional by their community and their doctors (Zhang et al. 2019, p. 7). Negative stigma has a direct impact on a schizophrenic's ability to thrive, despite their illness. One of the practical ways to combat this stigma is to take seriously the religious values of a schizophrenic person. This is especially important when it comes to the attitudes of mental health professionals. Negative, psychotic religious 
episodes can provide insight into the severity of a schizophrenic's symptoms, which can be important for determining how to proceed with treatment options. Positive religious episodes can allow schizophrenic individuals to make immense strides in their treatment, from feeling accepted in a religious community to using prayer to cope with difficult symptoms.

\section{Incorporating Religion into Treatment: The Risk/Benefit Ratio}

Another way to assess the claim that doctors should allow for spirituality and religion in therapy is to determine whether or not, "the expected benefits [of a treatment] outweigh the expected harms" (Greif and Šurkala 2020, p. 489). A therapy that has a "favorable risk/benefit ratio," "is in the patient's interest and it is rational for them to try it" (Greif and Surkala 2020, p. 489). Even if there are slightly better treatments available, if studies show that schizophrenics leave therapy if their religion is not respected, than how can mental health professionals even try other treatments if their patients have left therapy? It seems as though spirituality should be a foundation to treating schizophrenics, even if only to get them to stay in therapy.

As established above, incorporating spirituality into therapy improves a variety of schizophrenic patients' symptoms, including making them more likely to seek treatment (Zhang et al. 2019, p. 10). If a schizophrenic leaves therapy because their religious beliefs are received negatively by a mental health professional, studies show that they are less likely to receive treatment again (Zhang et al. 2019, p. 4). Therefore, the benefits of incorporating a schizophrenic patient's spirituality or religion in therapy, such as a decrease in self-stigma, seem to outweigh the negatives, such as a schizophrenic's refusal to get treatment because they don't feel that their doctors can relate to their spirituality.

Perhaps, however, this risk/benefit ratio is not so black and white. Although studies prove that religion and spirituality foster positive outcomes for schizophrenic patients, what can be said about the interference of mental health professionals in such practices? As mentioned earlier, religion provides schizophrenic patients with a lot of benefits, such as a decreased risk of suicide. When mental health professionals work through spirituality with their patients, can it do more harm than good? There are several issues that need to be evaluated before this question can be answered and an accurate risk/benefit ratio can be determined.

\section{The Lack of Religious Training for Medical Professionals}

One of the concerns about allowing mental health professionals to incorporate their patients' spirituality into therapy is their lack of training. Psychologists receive "little to no training and dialogue concerning spirituality or religious issues" during their education (Gorsuch and Shafranske 1984, p. 238). Psychologists often rely on various learned "theories, systems, methods and techniques" in their practice, but they are not taught any techniques for dealing with their clients' spirituality (Gorsuch and Shafranske 1984, p. 240). The "lack of 
emphasis in training on the important role that religion plays in health care may result in a decrease in religious involvement and the development" and as a result the more training that a medical professional receives, the less religious they become (Hafizi et al. 2014, p. 1375). Therefore, not only does the education system fail to inform medical professionals about the important link between mental health and religion, but the more training medical professionals receive, the less they value religion.

This lack of education about spiritual and religious issues also have several concerning consequences. One of the consequences of trying to treat client's spirituality without proper training is that the "role of psychotherapist" can shift "into the role of the clergy" when they practice spiritual techniques, which already "fall outside the expertise of most psychologists" (Barnett 2016, p. 8). Psychologists are also less religious than the general public (Gorsuch and Shafranske 1984, p. 232). If they are not trained to deal with religion and have no spirituality of their own, then how can they be expected to properly address their clients' spiritual needs?

\section{Religious Bias from Medical Professionals}

Even if a psychologist does have beliefs of their own, what if their beliefs contradict their clients'? Look at the case study of a woman named Donna, who sought out psychotherapy (in part) to deal with her relationship with her religion (Tummala-Narra 2009, p. 92). Donna was a Christian and asked her therapist, who was a Hindu, what her beliefs were (Tummala-Narra 2009, p. 91). Her therapist worried that their religious differences may negatively alter Donna's ability to share her religious life, including details about her church, as well as have the potential to impact "power dynamics" in their relationship (Tummala-Narra 2009, p. 92). Although the therapist's religious "disclosure contributed to a more open and authentic engagement with" Donna, this was mostly because Donna became more "comfortable" when she realized her therapist knew "about God" (TummalaNarra 2009, p. 91).

Several questions can be raised from the relationship between Donna and her therapist: Would Donna have become less comfortable if her therapist was an atheist? Would Donna have benefitted from the therapy more if her therapist was also a Christian and could therefore prescribe religion-specific treatments, such as comforting Bible verses? Even if the differences in religion didn't negatively impact Donna's relationship with her therapist - for example she was still able to open up about her religious life - that doesn't mean that this would be the case for all clients. These questions, therefore, are highly subjective and will differ from person to person. If, for example, a patient was uncomfortable opening up to a therapist who holds different beliefs, what then? The lack of training on the psychotherapist's part sure doesn't help.

Perhaps when the studies, such as the ones cited earlier, say that schizophrenics left therapy because they felt misunderstood by their therapists, the misunderstanding could be as simple as a difference of religious beliefs. It's easy to tell psychotherapists that treating their patients' beliefs should "not be solely 
based on [the therapists'] personal religious or spiritual practices" but how do we ensure that this is followed, especially when therapists are not taught how to deal with spiritual matters and/or have opposite personal beliefs (Barnett 2016, p. 7)?

This potential conflict of interest between a therapist's personal beliefs and their client's can become problematic because "clinical work is affected significantly by the psychologists' reliance upon their subjective experience as the template for understanding the client's phenomenal world" (Gorsuch and Shafranske 1984, p. 238). In other words, a therapist's personal beliefs have a direct impact on the way that they understand their client's belief systems. For example, when mental health professionals believe that schizophrenics are incapable of holding sound religious beliefs, they may paint all their schizophrenic patients' beliefs as irrational automatically. If they do this, they may fail to differentiate between psychotic delusions, which help them to understand their patient's state of mind, and religious experiences, which are helpful coping strategies.

This perception results in one of two outcomes: either the therapist sees their client's beliefs as "not relevant or [views them] as relevant and [understands them] within the framework of the therapist. In both instances, the therapeutic perspective is based on the personal, rather than the clinical orientation of the psychologist" (Gorsuch and Shafranske 1984, p. 239). This is not surprising considering the lack of therapists' psychological training in regards to spirituality.

\section{The Dangers of Incorporating Religious Interpretation into Therapy}

Schizophrenics already are stigmatized and are often seen as incapable of having valid religious beliefs (Sass 2001, p. 252). Therapists should not be tasked with interpreting religion in any specific way that goes beyond their training. Ideally, therapists would work alongside religious leaders as discussed later in the paper. It's best that therapists leave religion to spiritual leaders. Otherwise, therapists may view their client's religion through their own belief system. This could lead mental health professionals to further stigmatize their schizophrenic patients, who are already stigmatized enough (Gorsuch and Shafranske 1984, p. 239). For example, if " $35.2 \%$ of Chinese mental health professionals" have admitted to stigmatizing their patients, add on a diagnosis of schizophrenia (which is shown to be one of the more negatively stigmatized disorders in China) and then tell the therapist that they have to acknowledge the schizophrenic's religion, which could be the opposite of their own (Zhang et al. 2019, pp. 4-5). How would this not result in further stigmatization?

When therapists only see their patients' spirituality through the lens of their own belief systems, this can leave clients feeling like their own beliefs are not respected. As mentioned previously, this is one of the reasons that schizophrenics leave therapy and decline mentally as a result (Zhang et al. 2019, p. 4). It is also important to question, however, how much influence a therapists' personal beliefs can have over a vulnerable patient, such as someone who is suffering from a serious mental illness like schizophrenia. Instead of discovering their own identity through belief systems, such as religious communities, to find "meaning and 
connection with others," vulnerable patients may be swept up in the therapists' beliefs, especially if they see their therapist as a mentor or authority figure (Barnett 2016, p. 6).

The potential for a therapist to spiritually influence a patient seems especially plausible considering "the essential factor in the perception of spirituality as relevant within clinical work is the psychologist's personal stance towards spirituality" (Gorsuch and Shafranske 1984, p. 238). This means that whether the therapist will even discuss religion or not, to what degree and in what context depends largely on the therapists' own beliefs. Even if the therapist does not directly impose or even disclose their own beliefs, the way they interpret and thus discuss their patient's beliefs can still have a negative impact. For example, the therapist can use their personal beliefs to assign a truth value to their client's religion. A Buddist therapist, for example, may discourage their Christian client from going to church, encouraging them to meditate instead. As Barnett puts it, a therapist may erroneously ask themselves "this works for me so why not for my clients?" (Barnett 2016, p. 7). This could leave their patients with a feeling that their beliefs are wrong, which could result in negative consequences. For example, if one of their patients leaves their religious community, in which they found support.

\section{Bridging the Gap between Religion and Medicine}

"At this juncture, bridges are needed to cross the line between the traditional world which still governs the training of mental health workers and holistic psychological/social/mental/spiritual belief system so commonly endorsed by many patients" (Gopaul-McNicol 1997, p. 46). How can this gap be bridged? This paper will now focus on two commonly suggested solutions or "bridges": either train mental health professionals in religious therapy, which will be shown to be ineffective, or enact an interprofessional care system, an ideal partnership spanning across different medical and religious disciplines.

Starting with the former idea, if therapists want to explore spirituality with their clients, Barnett claims that it is important for them to "develop and maintain ongoing relationships with religious leaders or other experts on various faith traditions in our local communities" (Barnett 2016, p. 8). Barnett argues that understanding various religions is an ethics issue, which falls under the heading of "multicultural competence" (Barnett 2016, p. 6). As a result, "all mental health clinicians must be aware of, sensitive to, knowledgeable about, and respectful of all forms of diversity" (Barnett 2016, p. 6). He goes so far to argue that a therapist's refusal to bring spirituality into therapeutic discussion means that they have failed to provide "needed treatment services to those who are likely to seek [their] help" and are, therefore, unable to uphold the "minimally accepted ethics standards" of the profession (Barnett 2016, p. 9). Although he admits that there are times when therapists should refer clients to other spiritual leaders because "[therapists] each must also be cognizant of the limitations of [their] expertise," this is still quite a statement. 
Is it a therapist's ethical duty to talk to a client about religion, even if the therapist is uncomfortable discussing religion or is seriously lacking in religious expertise? What if such a discussion potentially results in a deeper stigmatization of more complicated mental illnesses, such as schizophrenia? Perhaps a line needs to be drawn between what a therapist is and isn't qualified to discuss before this question can be answered. This recommendation can be based off things such as the limitations of their training and studies about how they treat spirituality, such as only seeing their client's beliefs from the perspective of the therapist's own religion. This paper will now attempt to lay the groundwork for this much-needed exploration based on the small sampling of articles and research it has focused on thus far. Perhaps this will allow for future research to be conducted in this field.

Since therapists see their client's religion through the eyes of their personal beliefs, as discussed earlier, it is plausible that a Jewish therapist will learn Christianity through the eyes of Judaism, for example? Jews believe that Jesus was a phony, while Christians believe he is the son of God (Glazier 2020, p. 1). Will a Jewish therapist be comfortable (or even able) to not only appreciate their Christian client's viewpoint that Jesus is the son of God (which Jews disagree with), but to also prescribe Bible verses that encourage their client to seek peace in Jesus (Glazier 2020, p. 1)? Perhaps some therapists will, but if they can't they risk projecting their own beliefs onto the client. For example, a Jewish therapist with a strong faith may only choose verses from the Torah (part of what Christians call the "Old Testament" and the only part of the Bible that Jews follow), which leaves out over half of the Christian wisdom of the Bible (Glazier 2020, p. 1).

What should a therapist discuss in terms of religion? Donna from the case study, for example, talked to her therapist about things such as feeling like an "outsider" at her white church, but felt too afraid to attend a black church because "she had linked the black church with her father and his family" (Tummala-Narra 2009, p. 91). Since her father was a source of trauma for Donna, her therapist was well within her realm of expertise to help Donna process her trauma, which allowed Donna to explore her identity and find a new "religious community" that was more in line with Donna's beliefs (Tummala-Narra 2009, p. 93). This therapist did not require any in-depth knowledge of Donna's religion or the related religious texts to help Donna process her trauma related to her father, which gave Donna the confidence to explore her spirituality in different ways. In fact, the therapist had likely been trained on trauma during school and therefore was wellversed in the ways she could help Donna work through her trauma. Since the therapist did not impose her own beliefs nor did she try to instruct Donna as a religious leader would, such as providing her with Bible verses or providing biblically-based lessons about church diversity, this seems professionally reasonable. If Donna asked her therapist for specific religious advice, such as Bible verses, the therapist should refer her to a religious leader who can better answer Donna's questions. To give such advice would be unethical, because it falls outside of the therapist's expertise and training and could harm the patient.

Barnett would disagree, but his suggestions go beyond a therapist's trained abilities and are potentially dangerous. For example, he believes that psychotherapists could "[recommend] specific passages of scripture for the client 
to read and contemplate or even [integrate] the use of prayer into psychotherapy sessions" (Barnett 2016, p. 8). This requires a vast amount of self-taught religious expertise, since therapists receive no religious training. Some religious leaders devote their lives to learning how best to prescribe prayer and religious scripture to people within their religion. To expect a therapist to be well-versed enough in a variety of religion beliefs, cultures and holy texts in order to prescribe spiritually relevant ideas to their clients would not only pose an unrealistic and timeconsuming burden on therapists, but it could also backfire if they misunderstand the culture or texts, which could negatively impact clients. Refusing to acknowledge a patient's religious ideology can lead to suicide and psychosis, among other serious symptoms (Prout et al. 2016, p. 6). Therefore, providing therapists with religious training - which goes beyond their expertise-is not advisable. Therapists should, however, understand and respect when therapeutic discussions fall outside their realm of expertise. They should try to redirect the conversation back to topics within their expertise, such as the example in the previous paragraph about Donna, and/or refer their clients to religious experts.

As mentioned earlier, the additional stigmatism related to schizophrenics doesn't help. Mental health professionals already stigmatize people with schizophrenia and some even believe that their religious beliefs are invalid because they are not in their right mind (Sass 2001, p. 252). Many people who are deeply religious already think that other people's beliefs are wrong, such as when religious people claim that people from other religions are going to hell. How can we assume that religious mental health professionals will be any more tolerant (or worse more intolerant) towards people with a major mental illness such as schizophrenia?

Perhaps it is best for therapists to refer their clients to spiritual leaders when their clients ask for religion-specific advice, such as holy verses, to avoid acting as an unofficial clergy of a religion they may or may not have any personal experience with. The therapist can work through traumas or feelings that a client connects to the church, such as Donna's trauma with her father, but therapists should not try to prescribe holy verses or religion-specific advice outside of their own religion.

If a therapist tries to give advice that is best parsed from the mouth of a religious leader, such as what verses to read or meditations to practice, then they also rob their client of the opportunity to further bond with their own religious community. As mentioned earlier, one of the benefits of religion on a schizophrenic's mental wellbeing is their connection to an accepting community. By encouraging a patient to speak with their religious community about very specific religious advice, the therapist has helped the client delve deeper into their community. This allows the client to further develop positive coping strategies outside therapy. This gives the client more coping strategies to use instead of making them dependent on their therapist for their spiritual and mental wellbeing. 


\section{A Potential Solution: The Archstone Foundation's Interprofessional Care System}

Spreading out different responsibilities to a variety of professionals is a large facet of the some interprofessional care systems, such as the method advocated by the Archstone Foundation of Long Beach, California (Ferrell et al. 2009, p. 1). The interprofessional care system, mainly the one posed by the Archstone Foundation, is the second solution that this paper will focus on in regards to creating a safe space where schizophrenics can explore their spiritual beliefs. The Archstone Foundation's interprofessional care model posits "that spirituality or reflection should be part of a clinician's professional development," but asserts that patients should have a team of professionals who work together to satisfy the whole person and all of their needs (Balboni et al. 2014, p. 1588). This care system plants their roots in the "World Health Organization's definition of whole health, which defines health as the 'dynamic state of complete physical, mental, spiritual, and social well-being and not just the absence of disease or infirmity" (Balboni et al. 2014, p. 1587).

In the Archstone Foundation's interprofessional care model, a schizophrenic patient may go to a psychiatrist for medication, a psychotherapist for talk therapy and a religious leader for spiritual guidance, for example. Instead of being separate entities, which are often seen in a hierarchy with psychiatrists and medication being at the top, every person is seen as "equal members of the healthcare team" (Balboni et al. 2014, p. 1596). This gives each professional an "equal opportunity to shape their own 'plausibility structures' and their particular practices related to patient care (Balboni et al. 2014, p. 1594).

An example of how a healthcare team can work together can be seen as follows: "a chaplain may address physical pain but look to the physician or nurse on the team to deal with determining the cause of the pain and its treatment. Similarly, physicians, nurses, and others may identify and even diagnosis spiritual distress as well as identify spiritual resources of strength of the patient, while the Board Certified Chaplain (BCC) is the expert who can further evaluate the spiritual issues and recommend how to treat spiritual distress" (Balboni et al. 2014, p. 1588). This is especially beneficial because medical professionals aren't automatically trained in religious issues and "there is no standard uniform training among clergy as it relates to counseling the mentally challenged" (Dempsey et al., 2016, p. 76). Therefore, in this healthcare model each professional plays to their unique strengths and training to best serve the patient.

Another benefit of the Archstone Foundation's interprofessional care structure is that the professional connections are often pre-established. That way a person with a severe mental illness, such as schizophrenia, won't have to find, apply for and secure a wide variety of mental health professionals and community supports to aid in their recovery on their own.

These pre-established connections also assist mental health professionals by removing the burden of having to find relevant spiritual leaders or learning a new religion on their own. This interprofessional care structure ensures that a therapist will not have to act as a spiritual leader - which can be damaging to patientsallowing mental health professionals to focus on what they have been trained to 
do. This is important considering that many mental health professionals have no religious training. Even finding a relevant spiritual leader to consult may be difficult when a therapist has no experience in the religion and is therefore unaware of the different sects within religious communities, which can be challenging to navigate.

A schizophrenic patient, who may be wary of receiving treatment due to stigmatization, may take kindly to a medical team who respects religious leaders as equal contributors to their overall health. This care model promises to respect religious beliefs as equally important to bodily woes, such as physical pain. This means that schizophrenics will not have to fight to have their religious beliefs respected by their doctors, because the participating healthcare professionals have already committed to respecting religious beliefs as an important part of overall health (Balboni et al. 2014, p. 1588).

\section{The Mistakes Some Interprofessional Care Models Make}

Unfortunately, Ontario's version of interprofessional care, put out by the Ontario Ministry of Health and Long-Term Care's HealthForceOntario, does not mention anything about connecting religious or spiritual leaders, places of worship or religious community supports to healthcare workers (Poldre and Schleifer Taylor 2010). The focus, instead, is to educate different service providers on other services available, such as connecting community supports-which help people find employment - to homeless shelters, as well as to make information easily accessible for citizens on one central database (Poldre and Schleifer Taylor 2010, p. 9). It is a shame that spiritual leaders are not recognized as valid and equal parts of Ontario's criterion for interprofessional care partnership.

Supporters of the interprofessional care model posed by the Archstone Foundation, argue that it is a physician's "ethical obligation to attend to the psychosocial, spiritual, and existential distress of patients" (Balboni et al. 2014, p. 1587). By not including spirituality in their interprofessional care model, it could be argued that Ontario's interprofessional care model has failed to meet their patients' spiritual needs and is thus unethical.

For Ontario to leave spirituality out of their interprofessional care model is to ignore the fact that, "up to $80 \%$ " schizophrenics use religion as a coping strategy and if their beliefs are not respected, then this can result in major consequences, including an increased chance of suicide or the refusal to continue treatment (Chakrabarti et al. 2014, pp. 119, 121). To willfully leave out spiritual leaders from an interprofessional care proposal is to also ignore the proven positive benefits of religion on people with mental illnesses such as schizophrenia. This also robs health care professionals of the opportunity to develop official partnerships and relationships with diverse religious leaders. As mentioned previously, navigating the wide variety of religious beliefs is very difficult to do on one's own, especially with no formal training. Barnett would also argue that ignoring religion in healthcare fails to foster "multicultural competence," which is unethical (Barnett 2016, p. 6). 
Having an interprofessional care plan that includes partnerships with spiritual leaders not only helps professionals navigate their patient's diverse needs, but also validates spiritual leaders as an equal part of a patient's healthcare team. This validation is vital for people who are largely stigmatized, such as schizophrenics, and allows them to be treated in a way that respects them and their beliefs as individuals.

\section{The Risk/Reward Ratio of Interprofessional Care Models}

In light of this further exploration of the consequences and benefits of incorporating spirituality and religion into therapy, can it be said that benefits of such a practice outweigh the harms? Asking therapists to solely shoulder their patient's spiritual needs is unrealistic, especially without mandated, professional training. This burden also poses potential negative consequences for their patients, such as being told their beliefs are irrelevant when seen through the eyes of their therapist's personal beliefs (Gorsuch and Shafranske 1984, p. 239). It is difficult to accurately calculate whether or not leaving therapists to their own devices while navigating their schizophrenic patient's spirituality is more harmful than refusing to include spirituality at all. Regardless, asking a health professional to assess their patient's religion without any training seems quite dangerous. Society does not allow family doctors to preform open heart surgery, so why should they allow therapists - untrained in spiritual matters - to make sense of their patient's spiritual beliefs from the perspective of a religion that they may know nothing about?

The Archstone Foundation's interprofessional care model seems to have a promising "favorable risk/benefit ratio" as a useful therapy for people with schizophrenia (Greif and Šurkala 2020, p. 489). Many of the downsides that come with incorporating religion in therapy-such as the risk of mental health professionals stigmatizing or misinterpreting their patients' religion or their lack of training in religious matters - are greatly reduced because the cornerstone of this model relies on treating spiritual beliefs as equally important to physical ailments (Balboni et al. 2014, p. 1587). The benefits of religious practice for people with schizophrenia, such as community support, can be fully realized in an interprofessional care model that encourages religious or spiritual coping mechanisms. Therefore, the Archstone Foundation's interprofessional care model seems to be a great treatment option, giving people with schizophrenia a safe place to discuss their religious and spiritual beliefs. Since "positive religious coping" strategies are "the primary predictor of psychological well-being" in patients with schizophrenia, the benefits of this model seem to outweigh the risks if patients are able to use their healthcare team to develop and maintain positive religious coping strategies (Chakrabarti et al. 2014, p. 122). 


\section{Conclusion}

Above all, people with schizophrenia are people too with feelings, hardships, values and beliefs. Perhaps some of their symptoms may go beyond what many people will be able to comprehend as outsiders, but this is no reason to treat them with disrespect or ignore their beliefs because of their diagnosis. Validating their lived experiences - especially religious experiences which are so important to many people with schizophrenia - is vital for treatment. There are a variety of ways to encourage healthcare workers and the community to validate the importance of schizophrenic patients' religious beliefs. For example, stigma can be decreased by encouraging interprofessional care models that value spirituality, which sets the tone that religious beliefs are equally as important as other aspects of health, such as physical wellbeing. Such partnerships can also help healthcare workers better navigate the religious communities, which schizophrenics greatly benefit from participating in. As the Archstone Foundation writes "healing refers to the whole person and to how that person finds peace; a sense of coherence, solace, and meaning" (Balboni et al. 2014, p. 1589). For many, healing involves religious experiences, which "are a uniquely intimate communication and are worthy of diagnostic consideration and respect" (Allen and Wootton 1983, p. 219). What a simple way to better the lives of those suffering from a complicated illness.

\section{References}

Allen D, Wootton R (1983) Dramatic religious conversion and schizophrenic decompensation. Journal of Religion and Health 22(3): 212-220.

Balboni MJ, Peteet J, Puchalski C (2014) The relationship between medicine, spirituality and religion: three models for integration. Journal of Religion and Health 53(5): $1586-1598$.

Barnett JE (2016) Are religion and spirituality of relevance in psychotherapy? Spirituality in Clinical Practice 3(1): 5-9.

Biblica (n.d.) Matthew 1 - New international version (NIV). Biblica.

Cerully J, Collins R, Wong E (2016) Racial and ethnic differences in mental illness stigma and discrimination among Californians experiencing mental health challenges. RAND Corporation.

Chakrabarti S, Davuluri T, Grover S (2014) Religion, spirituality, and schizophrenia: a review. Indian Journal of Psychological Medicine 36(2): 119-124.

Dempsey K, Butler K, LaTrece G (2016) Black churches and mental health professionals: can this collaboration work? Journal of Black Studies 47(1): 73-87.

Escamilla R, Fresán A, López-Luna S, Páez F, Robles-García R (2014) History of religious delusions and psychosocial functioning among Mexican patients with paranoid schizophrenia. Journal of Religion and Health 53(6): 1622-1633.

Ferrell B, Otis-Green Puchalski C, Virani, R (2009) Improving the quality of spiritual care as a dimension of palliative care: the report of the consensus conference. Journal of Palliative Medicine 12(10): 885-904.

Glazier JS (2020) What are the main differences between a Jew and a Christian? Reform Judaism. 
Gopaul-McNicol S-A (1997) The role of religion in psychotherapy: a cross-cultural examination. Journal of Contemporary Psychotherapy 27(1): 37-48.

Gorsuch RL, Shafranske E (1984) Factors associated with the perception of spirituality in psychotherapy. The Journal of Transpersonal Psychology 16(2): 231-241.

Greif A, Surkala M (2020) Compassionate use of psychedelics. Medicine, Health Care and Philosophy 23(27): 485-496.

Hafizi S, Koenig HG, Arbabi M, Pakrah M, Saghazadeh A (2014) Attitudes of Muslim physicians and nurses toward religious issues. Journal of Religion and Health 53(5): 1374-1381.

$\mathrm{Ng} \mathrm{CH} \mathrm{(1997)} \mathrm{The} \mathrm{stigma} \mathrm{of} \mathrm{mental} \mathrm{illness} \mathrm{in} \mathrm{Asian} \mathrm{cultures.} \mathrm{Australian} \mathrm{and} \mathrm{New}$ Zealand Journal of Psychiatry 31(3): 382-390.

Poldre P, Schleifer Taylor J (2010) Implementing interprofessional care in Ontario final report of the interprofessional care strategic implementation committee. Toronto: HealfhForceOntario.

Prout TA, Ottaviano P, Taveras A, Sepulveda C, Torres J (2016) Parental and God representations among individuals with psychosis: a grounded theory analysis. Journal of Religion and Health 55(6): 2141-2153.

Sass LA (2001) Self and world in schizophrenia: three classic approaches. Philosophy, Psychiatry, \& Psychology 8(4): 251-270.

Tummala-Narra P (2009) The relevance of psychoanalytic perspective in exploring religious and spiritual identity in psychotherapy. Psychoanalytic Psychology 26(1): 83-95.

Zhang Z, Sun K, Jatchavala C, Koh J, Chia Y, Bose J, et al. (2019) Overview of stigma against psychiatric illnesses and advancements of anti-stigma activities in six asian societies. International Journal of Environmental Research and Public Health 17(1): 280. 
